\title{
Electrospray Post-Ionization Mass Spectrometry of Electrosurgical Aerosols
}

\author{
Sabine Guenther, ${ }^{1}$ Karl-Christian Schäfer, ${ }^{1}$ Júlia Balog, ${ }^{2}$ Júlia Dénes, ${ }^{1}$ Tamás Majoros, ${ }^{2}$ \\ Katalin Albrecht, ${ }^{2}$ Miklós Tóth, ${ }^{3}$ Bernhard Spengler, ${ }^{1}$ Zoltán Takáts ${ }^{1,2,4}$ \\ ${ }^{1}$ Institute for Inorganic and Analytical Chemistry, Justus Liebig University, Schubertstrasse 60, Haus 16, 35392 Giessen, \\ Germany \\ ${ }^{2}$ Medimass Ltd., Budapest, Hungary \\ ${ }^{3}$ Department of Health Science and Sports Medicine, Semmelweis University, Budapest, Hungary \\ ${ }^{4}$ First Department of Pediatrics, Semmelweis University, Budapest, Hungary
}

\begin{abstract}
The feasibility of electrospray (ES) ionization of aerosols generated by electrosurgical disintegration methods was investigated. Although electrosurgery itself was demonstrated to produce gaseous ions, post-ionization methods were implemented to enhance the ion yield, especially in those cases when the ion current produced by the applied electrosurgical method is not sufficient for MS analysis. Post-ionization was implemented by mounting an ES emitter onto a Venturi pump, which is used for ion transfer. The effect of various parameters including geometry, high voltage setting, flow parameters, and solvent composition was investigated in detail. Experimental setups were optimized accordingly. ES post-ionization was found to yield spectra similar to those obtained by the REIMS technique, featuring predominantly lipid-type species. Signal enhancement was 20- to 50-fold compared with electrosurgical disintegration in positive mode, while no improvement was observed in negative mode. ES post-ionization was also demonstrated to allow the detection of non-lipid type species in the electrosurgical aerosol, including drug molecules. Since the tissue specificity of the MS data was preserved in the ES post-ionization setup, feasibility of tissue identification was demonstrated using different electrosurgical methods.
\end{abstract}

Key words: Mass spectrometry, Tissue analysis, Rapid evaporative ionization, Post-ionization, Phospholipids, Electrospray, Electrosurgery

\section{Introduction}

$I^{1}$ nvestigation of samples in their ambient environment has long been a challenge for mass spectrometry. The basic obstacles included the indispensable sample preparation, and also the incompatibility of ionization methods with the environment or the sample itself. The development of

Electronic supplementary material The online version of this article (doi:10.1007/s13361-011-0230-9) contains supplementary material, which is available to authorized users.

Correspondence to: Zoltán Takáts; e-mail: zoltan.takats@anorg.chemie.unigiessen.de "direct" or "ambient" ionization methods has lifted some of these constraints [1], however the sensitivity of these methods ranging from laser ablation/desorption to desorption electrospray is generally poor $[2,3]$. It has soon been recognized that various desorption ionization methods produce a significant amount of partially charged aerosol besides gas-phase molecular ions. In fact, a negligible percentage of the desorbed molecules forms individual gaseous ions, the majority is transferred to the gas phase as clusters with low (or zero) specific electric charging [4]. Laser desorption/laser ablation provides excellent desorption efficiency and particularly low primary ion yield, especially in the absence of matrix compounds [5]. Hence, post- 
desorption ionization (or simply post-ionization) of the desorbed species was implemented at first in conjunction with lasers, using an additional subsequent laser shot at different wavelength for the ionization of desorbed neutrals $[6,7]$. Corona discharge-type atmospheric pressure chemical ionization was also developed later in conjunction with atmospheric pressure laser desorption $[8,9]$. In this case, more than two orders of magnitude signal enhancement was reported.

Electrospray post-ionization was originally developed for the ESI-MS analysis of fluids not compatible with an ESI source due to high salt content. Since both the solvent and the sample were nebulized by electrospray and ultrasound, respectively, the technique was termed fused-droplet electrospray (FD-ES) $[10,11]$. In case of the electrospray-assisted laser desorption/ionization (ELDI) method [12, 13], a similar setup was used for the post-ionization of particles desorbed by a pulsed nitrogen laser. Electrospray post-ionization was also developed in combination with infrared laser ablation, termed laser assisted electrospray ionization (LAESI) [14, 15]. Although matrix-assisted laser desorption ionization provides considerably better ionization efficiency than that of simple LDI [16], this can still be enhanced by electrospray post-ionization $[17,18]$. Another similar method is extractive electrospray ionization (EESI), which is also applied both for the ESI-MS analysis of fluid samples [19, 20] and for the post-ionization of neutrals desorbed from solid surfaces [21, 22].

Rapid evaporative ionization mass spectrometry (REIMS) method has recently been described as a solution for the in vivo investigation of biological tissues [23, 24]. The method is based on the rapid heating of the samples, which results in thermal evaporation and also ionization of certain constituents. However, since the ionization efficiency of REIMS is poor, its application can only be justified in those cases where there is no alternative method, i.e. intact tissue analysis. Although this application of REIMS in conjunction with electrosurgery [25] has been successfully implemented, it still suffers from sensitivity issues, especially in positive ion mode. Furthermore, certain electrosurgical techniques including electrocoagulation, spray coagulation, or bipolar electrosurgery do not yield sufficient ion current for realtime tissue identification by mass spectrometry. Since these techniques are widely used at fine surgical manipulations [26], it is highly desirable to extend the application area of MS-based tissue identification to these methods.

In the present study, we have implemented electrospray post-ionization for the enhancement of the sensitivity of REIMS technique. The effect of ionization method parameters on ion yield was studied, and the feasibility of coupling electrocoagulation with on-line mass spectrometric analysis using electrospray post-ionization was also investigated.

\section{Experimental}

All experiments were performed using a LTQ Orbitrap Discovery mass spectrometer (Thermo Scientific GmbH,
Bremen, Germany). Tissue evaporation was carried out using a RadioSurg 2200 (Meyer-Haake, Wehrheim, Germany) electrosurgical unit. For spray coagulation experiment, an Erbotom ICC 300 (Erbe Elektromedizin, Tübingen, Germany) unit was used. Aerosols formed upon electrosurgical dissection were transferred to the distant mass spectrometer using a $2 \mathrm{~m}$ long, $1 / 8 \mathrm{in}$. outer diameter, 1/16 in. inner diameter PTFE tubing (Fluidflon PTFE tubing; LIQUID-scan GmbH Co. KG, Überlingen, Germany) and a Venturi pump (VAC 100; Veriflo, UK) device. The experimental setup was similar to the REIMS setup described earlier [24].

The electrospray capillary was led through the Venturi pump as it is depicted in Figure 1. The capillary tip was secured into central position relative to the Venturi housing by a thin fiber. Extrusion distance of electrospray capillary was generally set to $3-4 \mathrm{~mm}$ if it is not specified otherwise. High voltage of the electrospray source was varied in the range of $0-5 \mathrm{kV}$. Solvent flow was provided by the built-in syringe pump of the mass spectrometer. Flow rates were varied in the range of $0-25 \mu \mathrm{L} / \mathrm{min}$.

\section{Materials and Methods}

Methanol (Uvasol) and acetic acid (p.a, 100\%) were obtained from Merck KGaA (Darmstadt, Germany). Ammonium-hydroxide (30\%) and water (HPLC grade) were obtained from Sigma-Aldrich Chemie $\mathrm{GmbH}$ (Steinheim, Germany). Systematic testing was performed using foodgrade porcine organs. Human and canine tissue specimens were obtained from University of Debrecen (Hungary) after ethical clearance was granted for these experiments.

\section{Identification of Ionic Species}

Ionic species in the mass spectra were identified based on exact mass measurements and MS/MS fragmentation patterns. The assignment of the ionic species to specific lipids was done manually using a Microsoft Excel macro created in-house. This macro allows the calculation of mass-to-

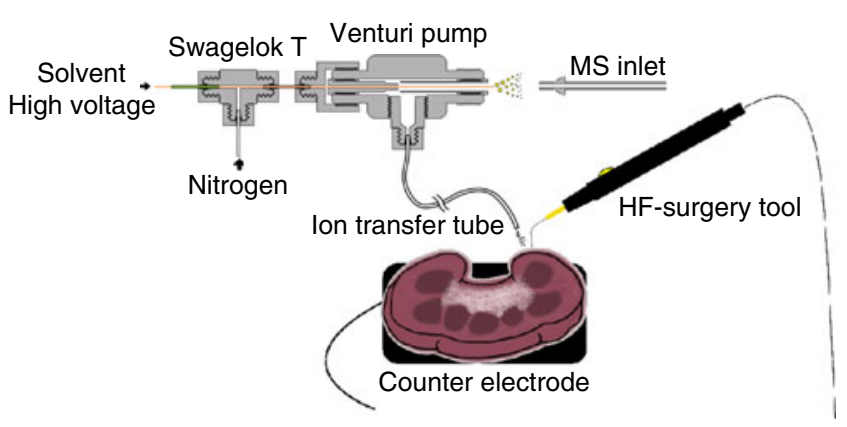

Figure 1. Scheme of the electrosurgical setup with ES post-ionization. The original REIMS setup was modified at the Venturi pump part in front of the MS inlet. A second Tpiece was added and a fused silica capillary is guided through the Venturi pump 
charge ratios for various ionic lipid species and the comparison with the measured masses. Characteristic fragment ionic species and neutral losses observed in the MS/ MS spectra are used to aid the lipid identification. In most of the cases MS/MS spectra of reference substances were obtained in order to verify the lipid species identification.

\section{Results and Discussion}

Data obtained by electrospray post-ionization of electrosurgical aerosol is shown in Figure 2 together with corresponding REIMS spectra.

As it is demonstrated, utilization of electrospray results in considerable signal enhancement in positive ion mode, with special emphasis on signals associated with structural phospholipids. While the REIMS spectra feature mainly thermal degradation products of various lipids in the mass range of 400-1000, electrospray post-ionization gives rise to ions corresponding to intact phospholipid species in the mass range of 600-900. In contrast, the intensity of negatively charged phospholipid ions shows a slight decrease, when ES post-ionization is implemented. The intensity of fatty acid carboxylate anions is significantly increased in the mass range of 150-350, however, the fatty acid distribution shows lower tissue specificity than that of phospholipids. Ionic species were identified based on exact mass measurements and MS/MS fragmentation patterns. The list of identified lipid species is presented in Table 1. As it is shown in the table, electrospray post-ionization enhances the formation of various adducts in positive ion mode. List of the identified ion species is given in Table S1 in details.

Spectral characteristics and intensities were found to be influenced by electrospray high voltage, source geometry, mass spectrometer potential settings, solvent flow rate, Venturi inlet pressure and the composition of the electrosprayed solvent system. The total ion current shows a similar voltage dependence in both positive and negative ion mode (Figure 3a) as observed in electrospray ionization experiments [27, 28]. When reaching the electrospray onset voltage (negative ion mode: $-1 \mathrm{kV}$, positive ion mode: 2 $\mathrm{kV}$ ) the total ion current starts to increase adding ions of the electrospray to the baseline current attributed to rapid evaporative ionization (REI) of the tissue [23]. At 3-4 kV a stable electrospray is obtained, resulting in no further increase of the total ion current with increasing voltage. In positive ion mode, phospholipid ion signal follows the trend
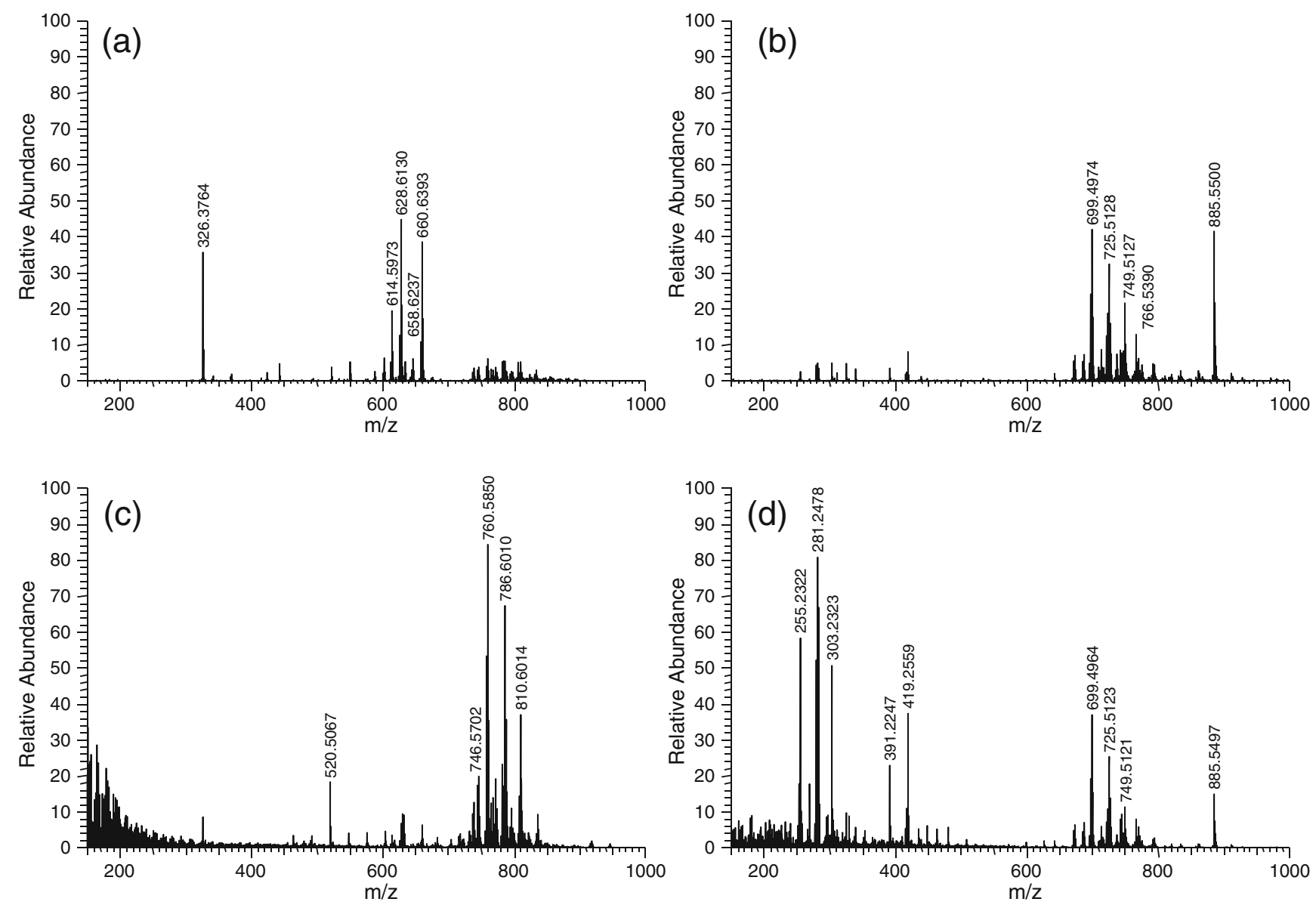

Figure 2. Mass spectra of porcine liver generated by REIMS in positive (a) and negative ion mode (b). Mass spectra of porcine liver generated by electrospray post-ionization REIMS in positive (c) and negative ion mode (d). All spectra are normalized to the same maximum intensity 
Table 1. List of identified lipids and degradation products generated by electrospray post-ionization REIMS in positive ion mode

Lipids and degradation products in positive mode, ES post-ionization

Diacylglycerols (DAG- $\left.\mathrm{H}_{2} \mathrm{O}\right) \quad 34: 0,34: 1,34: 2,36: 1,36: 2,36: 3,36: 4$

Phosphatidylethanolamines (PE) $\quad 34: 0,34: 1,34: 2,36: 1,36: 2,36: 3,36: 4$

$38: 1,38: 2,38: 3,38: 4,38: 5$ 40:2, 40:3,

$40: 4,40: 5,40: 6,42: 5,42: 6, \mathrm{p}-36: 4$,

p-38:4, p- $40: 5$

Phosphatidylcholines (PC) 32:0, 32:1, 34:0, 34:1, 34:2, 34:3, 34:4,

$36: 1,36: 2,36: 3,36: 4,38: 3,38: 4,38: 5$

$38: 6,40: 4,40: 5,40: 6,48: 2, \mathrm{p}-34: 4$,

p- $44: 2, \mathrm{p}-46: 2$

Phosphatic acids (PA)

Phosphatidylglycerols (PG)

Triaclyglycerols (TAG)

$\mathrm{NH}_{4}^{+}$adducts
$50: 1,50: 2$

$36: 0$

$48: 1,48: 2,49: 1,49: 2,49: 3,50: 0,50: 1$, $50: 2,50: 3,50: 4,51: 1,51: 2,51: 3,52: 1$ $52: 2,52: 3,52: 4,52: 3,53: 0,53: 1,53: 2$ $53: 3,54: 1,54: 2,54: 3,54: 4,54: 5,54: 6$, $55: 0,55: 1,55: 2,55: 3,55: 4,56: 1,56: 2$ $56: 3,56: 4,56: 5,56: 6,57: 2,57: 3,57: 4$ $57: 5,57: 6,58: 2,58: 3,58: 4,58: 5,58: 7$ $59: 3,59: 4,59: 5,59: 6,61: 6$
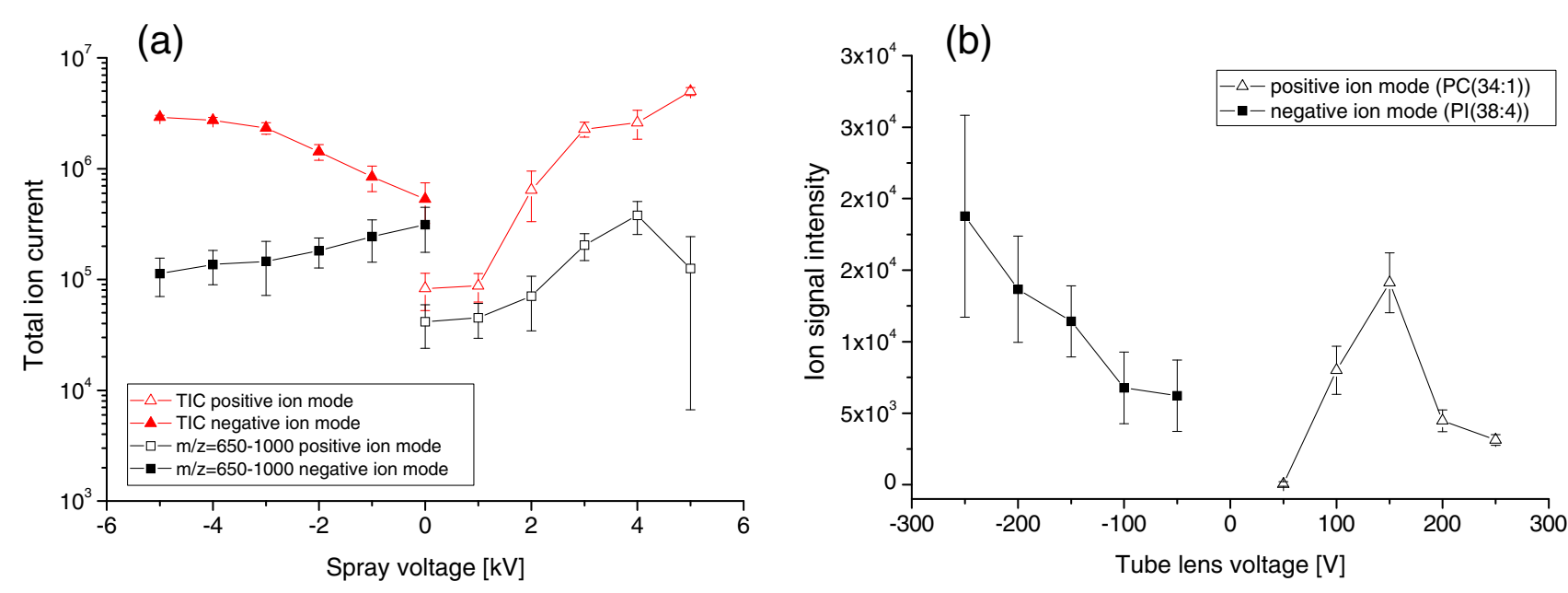

(c)

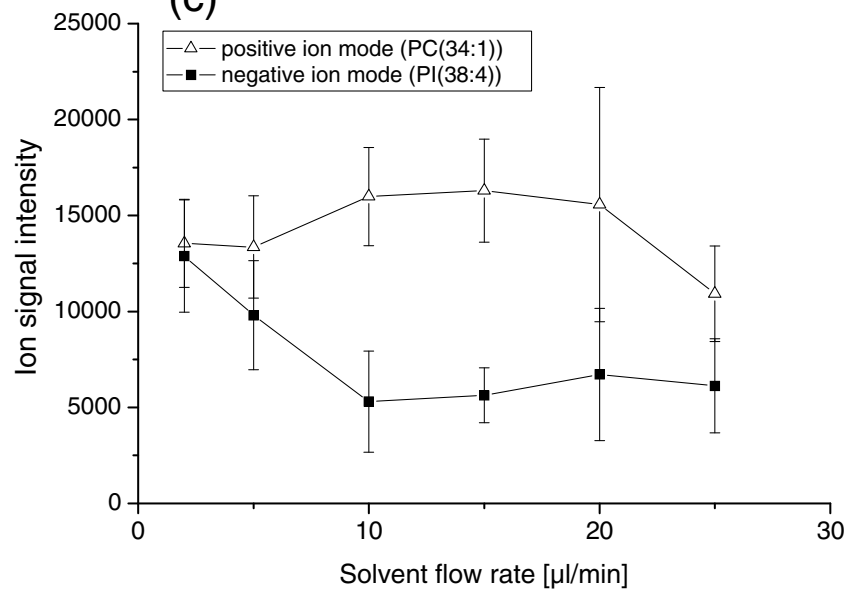

similar to total ion current, indicating a post-ionization of tissue-originated aerosol by the electrospray. However, the intensity of phospholipid anions shows different characteristics in negative mode. While there is a relatively high signal intensity at $0 \mathrm{~V}$ due to REI of phospholipids, this signal is apparently suppressed by the increasing electrospray background signals. The phenomenon was associated with the negatively charged droplets or clusters formed upon rapid evaporation of the tissue (vide infra).

Assuming that electrosurgical dissection results in boiling of the tissue due to the energy dissipated inside the bulk material, the boiling involving cavitation presumably leads to the formation of water vapor, gaseous thermal decomposition products, and aqueous droplets. Early investigations on aerosols generated by spraying or bubbling of aqueous liquids showed that the produced aerosols contain charged droplets of both polarities [29-32], and the size distributions are not identical in positive and negative modes (for further information see Supplementary material). We assume similar charged droplet formation processes during rapid evapora-

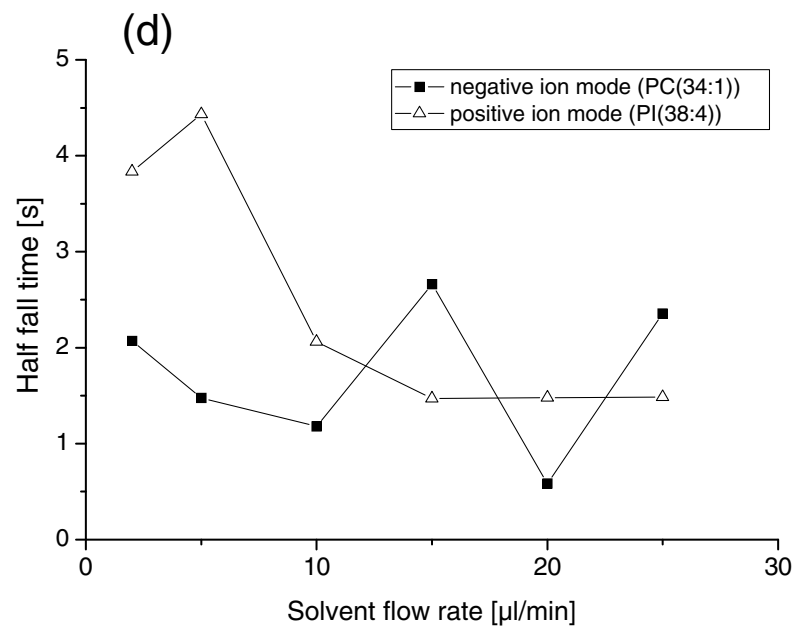

Figure 3. Dependence of overall signal intensity and intensity of phospholipid signal on electrospray high voltage (a) tube lens voltage (b) and solvent flow rate (c). (d) Depicts the dependence of half fall time of phospholipid ion signal intensity on electrospray solvent flow rate. All data are generated from porcine liver 
tion of biological tissues. The observations that positive ion mode electrospray post-ionization results in considerable signal enhancement regarding phospholipid ions and the negative ion mode electrospray post-ionization results in signal suppression strongly imply that phospholipid species are predominantly carried by negatively charged droplets. In case of electrospray post-ionization experiments, these negatively charged droplets will fuse only with positively charged electrospray droplets, which results in phospholipid signal enhancement in positive ion mode, and lack of fusion (due to Coulombic repulsion) results in signal suppression in negative ion mode.

As described in our earlier publications, REIMS phospholipid signals show limited or no dependence on tube lens voltage in case of Thermo instruments [24]. This observation was associated with the presence of large phospholipid clusters in the tube lens-skimmer region, which dissociate to individual ions in the first multipole ion guide. A scheme of this region of the atmospheric interface is shown in Figure S1. In contrast, positive ion mode electrospray post-ionization shows a similar tube lens voltage dependence as observed in electrospray experiments (Figure 3b, Figures S2 and S3, for further information on tube lens potential dependence of signal intensities see Supplementary material). In this case, the observed ions are presumably formed via electrospray ionization at atmospheric pressure, thus the tube lens potential shows the expected optimum phenomenon. Tube lens voltage dependence of the signal in negative ion mode electrospray post-ionization was similar to that of REIMS. While solvent flow rate had no significant effect on signal intensities (Figure 3c), it certainly affected the signal fall times. Half fall time was defined as the time interval necessary for the signal to fall to $50 \%$ when aerosol production is ceased. This value is crucially important from the point of view of surgical applications, since the signal corresponding to one tissue type should virtually immediately disappear when dissection line crosses the histologic border. Data shown on Figure 3d clearly indicate that solvent flow rates higher than $15 \mu \mathrm{L} / \mathrm{min}$ are suitable for surgical applications using the described experimental setup.

ESI solvent composition shows an optimum behavior both for organic solvent (methanol) and acidifying agent (acetic acid) concentration in positive ion mode ES post-ionization. Optimal phospholipid signal intensity was obtained using methanol/water $1: 1$ with $0.1 \%$ acetic acid. It has to be noted that optimal solvent composition is different for different compound classes, e.g. triglycerides require $90 \%$ methanol and $1 \%$ ammonium acetate for optimal ionization efficiency.

Positive ion mode phospholipid pattern obtained by ES post-ionization shows high histologic specificity, similarly to phospholipid patterns obtained by MALDI [33-35], DESI $[36,37]$ or REIMS [23, 24]. Tissue specificity is illustrated by principal component analysis (PCA) of data obtained from different porcine tissue specimens (Figure 4a.). While three-dimensional PCA provides only a demonstration of the feasibility of identification, a more sophisticated statistical (a)

$$
\begin{aligned}
& \text { Porcine liver } \\
& \text { Porcine lung alveolar tissue } \\
& \text { Porcine kidney cortex } \\
& \text { - Porcine kidney medulla } \\
& \text { Porcine kidney pelvis }
\end{aligned}
$$

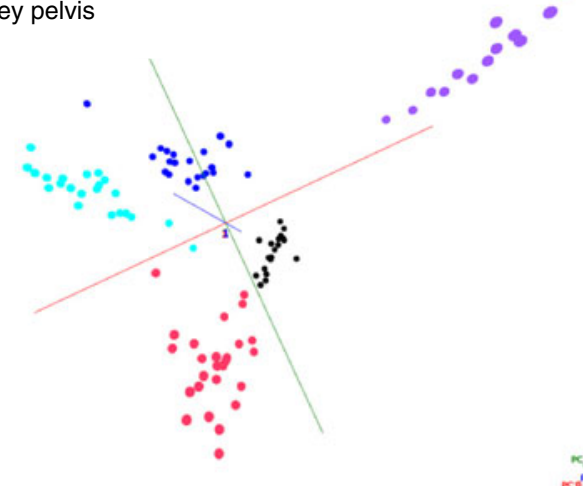

(b)

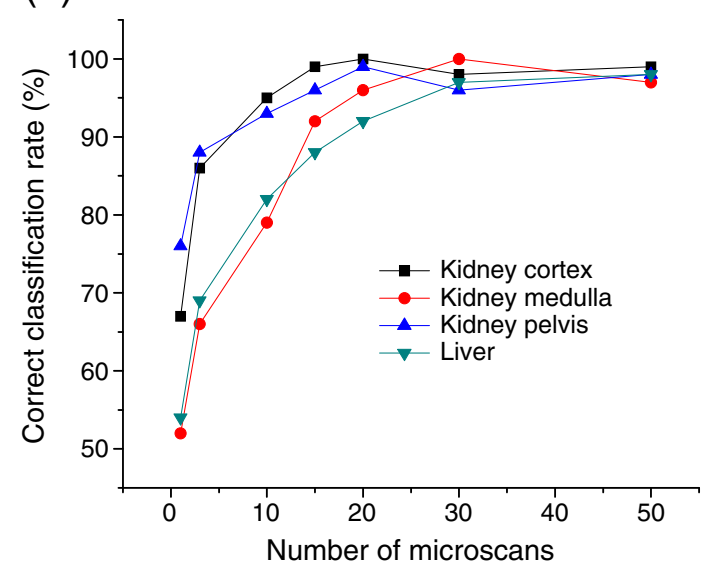

Figure 4. (a) PCA plot of five different porcine tissue types. Using three principle components the tissue types are clearly separated. (b) Accuracy of identification in dependence of the number of microscans averaged for identification

evaluation was also implemented for the identification of unknown tissue features.

The identification method, which was described earlier [24], utilizes a histologically assigned spectral database and combination of PCA and linear discriminant analysis (LDA) methods. Briefly, the real time identification procedure is started by selecting relevant spectra from the spectral database (e.g., all spectra corresponding to porcine kidney if porcine kidney is analyzed), performing a transformation on these data by PCA to 60 dimensions and modifying this data space by LDA to maximize the separation among different data groups. The result is a still 60 dimensional, but non-orthogonal data space with assigned regions for different histological groups. During the analysis of unknown samples, the actual data point is positioned in the data space and Mahalanobis distances are calculated from the centers of histologic groups. The data point is classified into the closest group using a certain threshold value, which depends on the average distance of database entries from the centre. Since 
the data space calculation is performed prior to analysis, the identification can be done in quasi-realtime $(<1 \mathrm{~s})$. Identification performance for different tissue types using positive ion mode ES post-ionization is shown in Figure 4b. Overall absolute sensitivity of identification is illustrated by a plot showing the dependence of accuracy of identification as a function of data acquisition time given in number of microscans.

Positive ion mode ES post-ionization has also been tested for the analysis of electrosurgical aerosol formed on the forced fulgurative coagulation of tissues. Electrosurgical instruments generally have two different monopolar application modes, viz., cutting mode and coagulation mode [25, 38, 39]. Most instruments utilize a constant amplitude sinus rf $\mathrm{AC}$ for cutting and attenuated waveform packets for coagulation as it is demonstrated in Figure S4. While cutting mode results in boiling of the tissue due to the energy dissipated inside the bulk material, coagulation mode involves extensive rf discharge, which evaporates material from the tissue surface at high temperatures. The underlying mechanistic difference is that "boiling" involves the disintegration (atomization) of tissue material yielding an aerosol, while "surface evaporation" comprises the desorption of molecular species from the gas/solid interface. As a result, cutting mode results in the formation of tissue originated molecular ions (REIMS) (Figure 5a), while coagulation mode produces only low molecular weight $(\mathrm{m} / \mathrm{z}<100)$ unspecific fragment ions. Since surgeons often use coagulation mode for fine preparations, there was a strong need to provide real-time tissue identification data in these cases. Positive ion mode electrospray post-ionization spectra using coagulation mode are shown in Figure 5b. As data clearly show, there is no significant difference between cutting and coagulation modes when ES post-ionization is used, indicating that the composition of the neutral aerosol is similar using the two modes.

Bipolar electrosurgery is another method for electrosurgical tissue evaporation and coagulation $[26,40]$. In this case, the surgical handpiece contains both electrodes in the form of so-called bipolar forceps. Tissue part (or blood
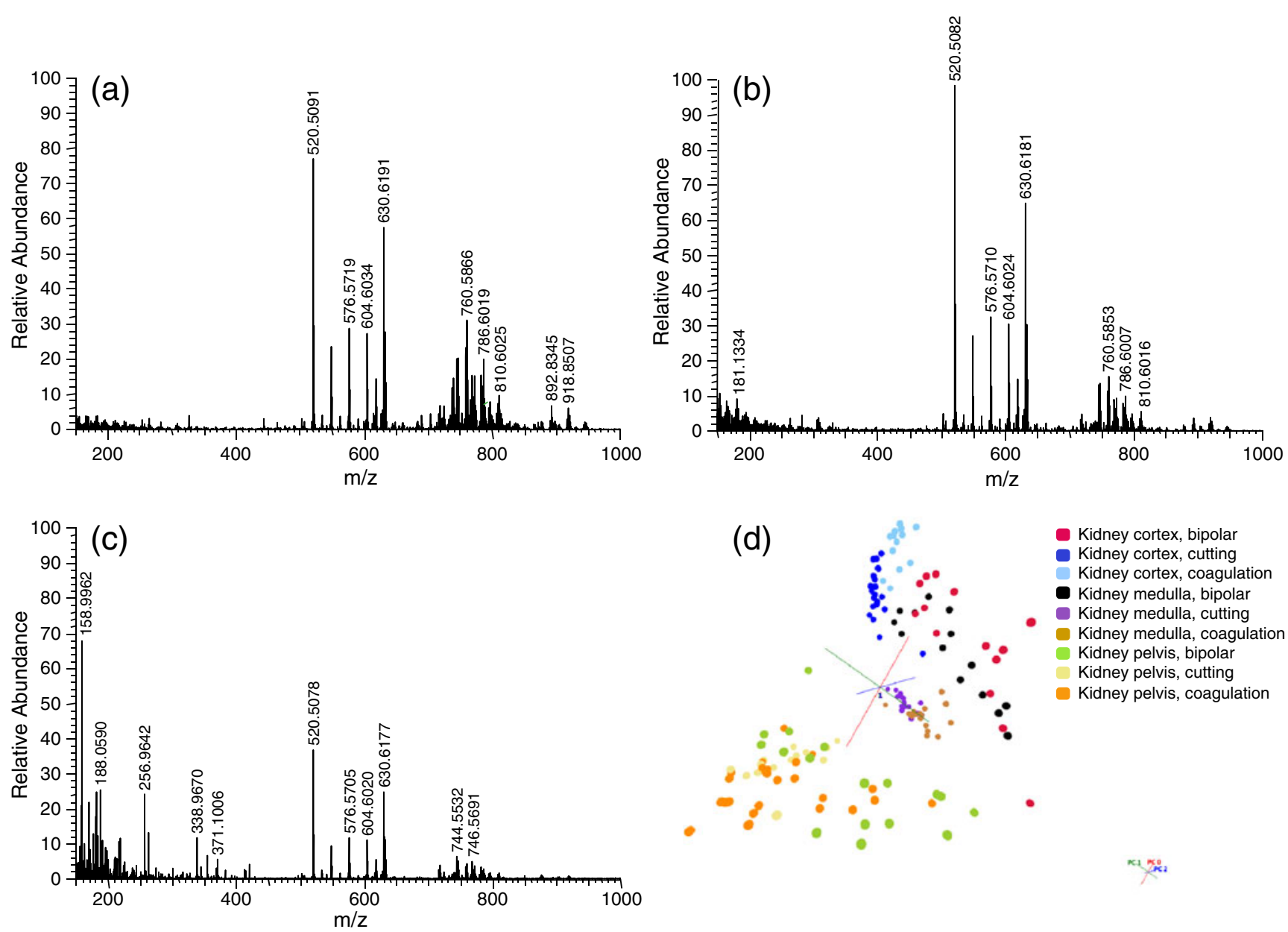

Figure 5. Mass spectra of porcine kidney (cortex) generated by positive ion mode electrospray post-ionization REIMS using the electrosurgical instrument in cutting mode (a), coagulation mode (b) and with bipolar forceps (c). All spectra are normalized. (d) Shows the three dimensional principal component analysis of different porcine kidney spectra obtained by the positive ion electrospray post-ionization of electrosurgical aerosols. The incomplete separation of method-specific data indicates high spectral similarity 
vessel) is grasped by the jaws of the forceps and rf current is applied between the jaws. This way only a minute amount of tissue is evaporated and the body of the patient does not become part of the electric circuit, both of which are critical, e.g., in case of brain surgery. Bipolar forceps are also used for fine preparations, thus in situ tissue identification in this case would also make a critical difference. However, bipolar tools give poor signal using the REIMS setup, due to sensitivity problems associated with the minor amount of evaporated material. Since the overall sensitivity of positive ion mode ES post-ionization is better than that of negative mode REIMS analysis, the technique was also tested in this case, yielding ion current with low, but still sufficient sensitivity (Figure $5 \mathrm{c}$ ).

Analysis of porcine kidney samples revealed that even three-dimensional PCA cannot fully differentiate between the cut and coagulation modes, and it provides only partial separation for bipolar mode as it is demonstrated on Figure 5d. Spectral similarity also implies that one common spectral library can be used for identification purposes in all cases of ES post-ionization experiments, regardless of the applied electrosurgical method. REIMS database cannot be directly utilized for tissue identification in ES-REIMS experiments due to the discussed spectral differences (Figure 2); hence, a separate database is needed for these experiments. However, there is a necessary correlation between the spectra generated by the different ionization approaches, since the detected species, the sample and, thus, the underlying chemical basis are the same in both cases. This correlation is currently evaluated and will hopefully allow the use of one spectral database for both measurement methods in the future.

Electrospray post-ionization also allows the detection of non-lipid species. The feasibility of the in vivo detection of drug molecules was tested on human tumor xenografts in SCID mouse. In principle, selectively binding drug molecules can be used for the in vivo chemical labeling of tumors, facilitating the mass spectrometric differentiation between tumor and healthy cells [41-43]. Molecularly targeted tyrosine kinase inhibitors and tyrosine kinase overexpressing tumors are ideal models to test the feasibility of this application. SCID mice carrying human HT-29 colon adenocarcinoma xenograft were administered with a single dose of imatinib, and mouse organs were analyzed postmortem using electrosurgery/ES post-ionization. Imatinib was detected successfully in the tumor tissue and the kidney of the animals (data are shown in Figure S5). Identification of imatinib was done by exact mass measurement and MS/ MS experiments.

\section{Conclusions}

The analysis of electrosurgical aerosol by electrospray postionization mass spectrometry is a versatile tissue identification method, which solves most of the sensitivity problems associated with the earlier described REIMS technology.
Besides providing means for the real-time monitoring of tissue specific positive ion mode phospholipid profiles, the method is also capable of the detection of low molecular weight metabolites and drug molecules. It was demonstrated, that the presented method gives uniform data independently from the electrosurgical method used, and makes the tracking of fine surgical preparations feasible by coupling coagulation and bipolar methods with on-line mass spectrometric detection.

The presented technology can directly be introduced into various areas of cancer surgery, including the resection of metastatic brain tumors or enucleative removal of renal cell carcinomas.

\section{Acknowledgments}

The authors acknowledge funding for this work by the European Research Council under the Starting Grant scheme (contract no. 210356) and Hessisches Ministerium für Wissenschaft und Kunst (LOEWE Schwerpunkt "AmbiProbe").

\section{References}

1. Van Berkel, G.J., Pasilis, S.P., Ovchinnikova, O.: Established and Emerging Atmospheric Pressure Surface Sampling/Ionization Techniques for Mass Spectrometry. J. Mass Spectrom. 43, 1161-1180 (2008)

2. Salter, T.L., Green, F.M., Gilmore, I.S., Seah, M.P., Stokes, P.: A Comparison of SIMS and DESI and Their Complementarities. Surf. Interface Anal. 43, 294-297 (2011)

3. Denes, J., Katona, M., Hosszu, A., Czuczy, N., Takats, Z.: Analysis of Biological Fluids by Direct Combination of Solid Phase Extraction and Desorption Electrospray Ionization Mass Spectrometry. Anal. Chem. 81, 1669-1675 (2009)

4. Mowry, C.D., Johnston, M.V.: Simultaneous Detection of Ions and Neutrals Produced by Matrix-Assisted Laser Desorption. Rapid Commun. Mass Spectrom. 7, 569-575 (1993)

5. Van Breemen, R.B., Snow, M., Cotter, R.J.: Time-Resolved Laser Desorption Mass Spectrometry. I. Desorption of Preformed Ions. Int. J. Mass Spectrom. Ion Processes 49, 35-50 (1983)

6. Spengler, B., Bahr, U., Karas, M., Hillenkamp, F.: Postionization of Laser-Desorbed Organic and Inorganic Compounds in a Time of Flight Mass Spectrometer. Anal. Instrum. 17, 173-193 (1988)

7. Dreisewerd, K., Schürenberg, M., Karas, M., Hillenkamp, F.: Influence of the Laser Intensity and Spot Size on the Desorption of Molecules and Ions in Matrix-Assisted Laser Desorption/Ionization with a Uniform Beam Profile. Int. J. Mass Spectrom. 141, 127-148 (1995)

8. Coon, J.J., McHale, K.J., Harrison, W.W.: Atmospheric Pressure Laser Desorption/Chemical Ionization Mass Spectrometry: A New Ionization Method Based on Existing Themes. Rapid Commun. Mass Spectrom. 16, 681-685 (2002)

9. Coon, J.J., Harrison, W.W.: Laser Desorption-Atmospheric Pressure Chemical Ionization Mass Spectrometry for the Analysis of Peptides from Aqueous Solutions. Anal. Chem. 74, 5600-5605 (2002)

10. Shiea, J., Chang, D.Y., Lin, C.H., Jiang, S.J.: Generating Multiply Charged Protein Ions by Ultrasonic Nebulization/Multiple ChannelElectrospray Ionization Mass Spectrometry. Anal. Chem. 73, 4983-87 (2001)

11. Shieh, I.F., Lee, C.Y., Shiea, J.: Eliminating the Interferences from TRIS Buffer and SDS in Protein Analysis by Fused-Droplet Electrospray Ionization Mass Spectrometry. J. Proteome Res. 4, 606-612 (2005)

12. Shiea, J., Huang, M.Z., Hsu, H.J., Lee, C.Y., Yuan, C.H., Beech, I., Sunner, J.: Electrospray-Assisted Laser Desorption/Ionization Mass Spectrometry for Direct Ambient Analysis of Solids. Rapid Commun. Mass Spectrom. 19, 3701-3704 (2005) 
13. Huang, M.Z., Jhang, S.S., Cheng, C.N., Cheng, S.C., Shiea, J.: Effects of Matrix, Electrospray Solution, and Laser Light on the Desorption and Ionization Mechanisms in Electrospray-Assisted Laser Desorption Ionization Mass Spectrometry. Analyst 135, 759-766 (2010)

14. Nemes, P., Vertes, A.: Laser Ablation Electrospray Ionization for Atmospheric Pressure, In Vivo, and Imaging Mass Spectrometry. Anal. Chem. 79, 8098-8106 (2007)

15. Nemes, P., Woods, A.S., Vertes, A.: Simultaneous Imaging of Small Metabolites and Lipids in Rat Brain Tissues at Atmospheric Pressure by Laser Ablation Electrospray Ionization Mass Spectrometry. Anal. Chem. 82, 982-988 (2010)

16. Karas, M., Bachmann, D., Bahr, U., Hillenkamp, F.: Matrix-Assisted Ultraviolet Laser Desorption of Non-Volatile Compounds. Int. J. Mass Spectrom. Ion Processes 78, 53-68 (1987)

17. Sampson, J.S., Hawkridge, A.M., Muddiman, D.C.: Generation and Detection of Multiply-Charged Peptides and Proteins by MatrixAssisted Laser Desorption Electrospray Ionization (MALDESI) Fourier Transform Ion Cyclotron Resonance Mass Spectrometry. J. Am. Soc. Mass. Spectrom. 17, 1712-1716 (2006)

18. Sampson, J.S., Hawkridge, A.M., Muddiman, D.C.: Development and Characterization of an Ionization Technique for Analysis of Biological Macromolecules: Liquid Matrix-Assisted Laser Desorption Electrospray Ionization. Anal. Chem. 80, 6773-6778 (2008)

19. Chen, H.W., Venter, A., Cooks, R. G.: Extractive Electrospray Ionization for Direct Analysis of Undiluted Urine, Milk, and Other Complex Mixtures Without Sample Preparation. Chem. Commun. 2042-2044 (2006)

20. Chingin, K., Gamez, G., Chen, H.W., Zhu, L., Zenobi, R.: Rapid Classification of Perfumes by Extractive Electrospray Ionization Mass Spectrometry (EESI-MS). Rapid Commun. Mass Spectrom. 22, 20092014 (2008)

21. Chen, H.W., Wortmann, A., Zenobi, R.: Neutral Desorption Sampling Coupled to Extractive Electrospray Ionization Mass Spectrometry for Rapid Differentiation of Bilosamples by Metabolomic Fingerprinting. $J$. Mass Spectrom. 42, 1123-1135 (2007)

22. Chen, H.W., Sun, Y.P., Wortmann, A., Gu, H.W., Zenobi, R.: Differentiation of Maturity and Quality of Fruit Using Noninvasive Extractive Electrospray Ionization Qquadrupole Time-of-Flight Mass Spectrometry. Anal. Chem. 79, 1447-1455 (2007)

23. Schäfer, K.C., Denes, J., Albrecht, K., Szaniszlo, T., Balog, J., Skoumal, R., Katona, M., Toth, M., Balogh, L., Takats, Z.: In Vivo, In Situ Tissue Analysis Using Rapid Evaporative Ionization Mass Spectrometry. Angew. Chem. Int. Ed. 48, 8240-8242 (2009)

24. Balog, J., Szaniszlo, T., Schaefer, K.C., Denes, J., Lopata, A., Godorhazy, L., Szalay, D., Balogh, L., Sasi-Szabo, L., Toth, M., Takats, Z.: Identification of Biological Tissues by Rapid Evaporative Ionization Mass Spectrometry. Anal. Chem. 82, 7343-7350 (2010)

25. Massarweh, N.N., Cosgriff, N., Slakey, D.P.: Electrosurgery: History, Principles, and Current and Future Uses. J. Am. Coll. Surg. 202, 520$530(2006)$

26. Bulsara, K.R., Sukhla, S., Nimjee, S.M.: History of Bipolar Coagulation. Neurosurg. Rev. 29, 93-96 (2006)

27. Ikonomou, M.G., Blades, A.T., Kebarle, P.: Electrospray-Ion Spray: A Comparison of Mechanisms and Performance. Anal. Chem. 63, 1989$1998(1991)$
28. Wampler, F., Blades, A., Kebarle, P.: Negative Ion Electrospray Mass Spectrometry of Nucleotides: Ionization from Water Solution with $\mathrm{SF}_{6}$ Discharge Suppression. J. Am. Soc. Mass. Spectrom. 4, 289-295 (1993)

29. Chapman, S.: Carrier Mobility Spectra of Spray Electrified Liquids. Phys. Rev. 52, 184 (1937)

30. Chapman, S.: Carrier Mobility Spectra of Liquids Electrified by Bubbling. Phys. Rev. 54, 520 (1938)

31. Chapman, S.: Interpretation of Carrier Mobility Spectra of Liquids Electrified by Bubbling and Spraying. Phys. Rev. 54, 528 (1938)

32. Dodd, E.E.: The Statistics of Liquid Spray and Dust Electrification by the Hopper and Laby Method. J. Appl. Phys. 24, 73-80 (1953)

33. Trim, P.J., Atkinson, S.J., Princivalle, A.P., Marshall, P.S., West, A., Clench, M.R.: Matrix-Assisted Laser Desorption/Ionization Mass Spectrometry Imaging of Lipids in Rat Brain Tissue with Integrated Unsupervised and Supervised Multivariant Statistical Analysis. Rapid Commun. Mass Spectrom. 22, 1503-1509 (2008)

34. Ishida, Y., Madonna, A.J., Rees, J.C., Meetani, M.A., Voorhees, K.J.: Rapid Analysis of Intact Phospholipids from Whole Bacterial Cells by Matrix-Assisted Laser Desorption/Ionization Mass Spectrometry Combined with On-Probe Sample Pretreatment. Rapid Commun. Mass Spectrom. 16, 1877-1882 (2002)

35. Römpp, A., Guenther, S., Schober, Y., Schulz, O., Takats, Z., Kummer, W., Spengler, B.: Histology by Mass Spectrometry: Label-Free Tissue Characterization Obtained from High-Accuracy Bioanalytical Imaging. Angew. Chem. Int. Ed. 49, 3834-3838 (2010)

36. Dill, A.L., Ifa, D.R., Manicke, N.E., Costa, A.B., Ramos-Vara, J.A., Knapp, D.W., Cooks, R.G.: Lipid Profiles of Canine Invasive Transitional Cell Carcinoma of the Urinary Bladder and Adjacent Normal Tissue by Desorption Electrospray Ionization Imaging Mass Spectrometry. Anal. Chem. 81, 8758-8764 (2009)

37. Eberlin, L.S., Dill, A.L., Costa, A.B., Ifa, D.R., Cheng, L., Masterson, T., Koch, M., Ratliff, T.L., Cooks, R.G.: Cholesterol Sulfate Imaging in Human Prostate Cancer Tissue by Desorption Electrospray Ionization Mass Spectrometry. Anal. Chem. 82, 3430$3434(2010)$

38. Chino, A., Karasawa, T., Uragami, N., Endo, Y., Takahashi, H., Fujita, R.: A Comparison of Depth of Tissue Injury Caused by Different Modes of Electrosurgical Current in a Pig Colon Model. Gastrointest. Endosc. 59, 374-379 (2004)

39. Wu, M.P., Ou, C.S., Chen, S.L., Yen, E.Y.T., Rowbotham, R.: Complications and Recommended Practices for Electrosurgery in Laparoscopy. Am. J. Surg. 179, 67-73 (2000)

40. Kennedy, J.S., Buysse, S.P., Lawes, K.R., Ryan, T.P.: Recent Innovations in Bipolar Electrosurgery. Minim. Invas. Ther. Allied Technol 8, 95-99 (1999)

41. Ross, J.S., Schenkein, D.P., Pietrusko, R., Rolfe, M., Linette, G.P., Stec, J., Stagliano, N.E., Ginsburg, G.S., Symmans, W.F., Pusztai, L., Hortobagyi, G.N.: Targeted Therapies for Cancer. Am. J. Clin. Pathol. 122, 598-609 (2004)

42. Druker, B.J.: Perspectives on the Development of Imatinib and the Future of Cancer Research. Nat. Med. 15, 1149-1152 (2009)

43. Collins, I., Workman, P.: New Approaches to Molecular Cancer Therapeutics. Nat. Chem. Biol. 2, 689-700 (2006) 